\title{
Incidence, mechanism and prognostic value of activated AKT in
}

\section{pancreas cancer}

\author{
MG Schlieman', BN Fahy', R Ramsamooj ${ }^{2}$, L Beckett ${ }^{3}$ and RJ Bold*,I \\ 'Department of Surgery, University of California Davis Medical Center, Sacramento, CA 95817, USA; ${ }^{2}$ Department of Pathology, University of California \\ Davis Medical Center, Sacramento, CA 95817, USA; ${ }^{3}$ Department of Epidemiology and Preventative Medicine, University of California Davis Medical \\ Center, Sacramento, CA 95817, USA
}

When activated, the serine/threonine kinase AKT mediates an antiapoptotic signal implicated in chemoresistance of various cancers. The mechanism(s) of AKT activation are unknown, though overexpression of HER-2/neu has been implicated in breast cancer. Therefore, we determined the incidence of activated AKT in human pancreatic cancer, whether HER-2/neu is involved in AKT activation, and if AKT activation is associated with biologic behaviour. HER-2/neu expression and AKT activation were examined in seven pancreatic cancer cell lines by Western blotting. The in vitro effect of HER-2/neu inhibition on AKT activation was similarly determined. Finally, 78 pancreatic cancer specimens were examined for AKT activation and HER-2/neu overexpression, and correlated with the clinical prognostic variable of histologic grade. HER-2/neu was overexpressed in two of seven cell lines; these two cell lines demonstrated the highest level of AKT activation. Inhibition of HER-2/neu reduced AKT activation in vitro. AKT was activated in 46 out of 78 (59\%) of the pancreatic cancers; HER-2/neu overexpression correlated with AKT activation $(P=0.015)$. Furthermore, AKT activation was correlated with higher histologic tumour grade $(P=0.047)$. Thus, it is concluded that AKT is frequently activated in pancreatic cancer; this antiapoptotic signal may be mediated by HER-2/neu overexpression. AKT activation is associated with tumour grade, an important prognostic factor.

British Journal of Cancer (2003) 89, 21 I0-21 15. doi:10.1038/sj.bjc.6601396 www.bjcancer.com

(c) 2003 Cancer Research UK

Keywords: pancreatic cancer; AKT; HER-2/neu

It is well established that part of the phenotype of cancer cells is resistance to apoptosis, though the cellular mechanisms that mediate this characteristic are not fully understood. AKT has been recently demonstrated to be a major mediator of survival signals in a variety of cells, including cancer cells (Tang et al, 2001; Vivanco and Sawyers, 2002). Constitutive activation of AKT provides a potent antiapoptotic signal in cancer cells conferring chemo- and radioresistance (Brognard et al, 2001; West et al, 2002). To date, there have been a few studies on the incidence of AKT activation in various tumours using archived pathologic specimens (Dhawan et al, 2002; Gupta et al, 2002; Itoh et al, 2002; Malik et al, 2002; Perez-Tenorio and Stal, 2002). While these reports correlate AKT activation with some biologic property of the tumour, none has examined potential upstream activating mechanisms. In prostate cancer, the AKT activation occurred in 33 out of 125 specimens and was more frequent in higher Gleason grade cancers (Malik et al, 2002); in colon cancer, the AKT activation was observed in 30 out of 65 tumours and associated with advanced clinicopathologic stage (Itoh et al, 2002); in breast cancer, the AKT activation was frequently observed (50 out of 93 ) and predicted a worse prognosis

* Correspondence: Dr RJ Bold, Division of Surgical Oncology, Suite 30 I0, UC Davis Cancer Center, 4501 X Street, Sacramento, CA 95817, USA; E-mail: richard.bold@ucdmc.ucdavis.edu

Received 3 June 2003; revised 19 August 2003; accepted 16 September 2003 in endocrine treated patients (Perez-Tenorio and Stal, 2002); in melanoma, the AKT activation was observed in eight out of 12 specimens and the incidence of AKT activation was more common than in precursor lesions (Dhawan et al, 2002); and in head and neck squamous cell carcinoma, the AKT activation was observed in 25 out of $38(66 \%)$ specimens and correlated with local recurrence (Gupta et al, 2002).

Pancreatic cancer is a highly lethal malignancy resistant to the apoptosis-inducing effects of radio- and chemotherapy (Bardeesy and DePinho, 2002; Heinemann, 2002). Recent reports have suggested that AKT, a target of phosphatidylinositol 3-kinase (PI3K), is phosphorylated and thus activated under basal conditions in a variety of pancreatic cancer cell lines, possibly contributing to the inherent apoptotic-resistance (Xue et al, 2000; Bondar et al, 2002; Samatar et al, 2002). These data come from the available cell lines, though no data are available on the incidence of AKT activation in human tumours. Recent reports have demonstrated that the AKT pathway is a potent survival signal in these pancreatic cancer cell lines, as inhibition of PI3K, the upstream activator of AKT, has been shown to sensitise these cells to the apoptotic effect of chemotherapy in vitro ( $\mathrm{Ng}$ et al, 2000; Perugini et al, 2000; Yao et al, 2002). Furthermore, using these cell lines in animal studies, inhibition of PI3K was well tolerated and increased the efficacy of chemotherapy in vivo ( $\mathrm{Ng}$ et al, 2000; Bondar et al, 2002). Whether AKT represents an important survival signal and is an appropriate target for molecular-based therapy awaits validation of the incidence and clinical consequence of AKT activation in pancreatic cancer specimens. 
There has been intense investigation into the potential upstream events that mediate activation of AKT in cancer cells. Various mediators that have been identified are PTEN (Haas-Kogan et al, 1998), K-ras (Guerrero et al, 2000), and HER-2/neu (Zhou et al, 2001), though other receptor tyrosine kinases have also been shown to be involved in AKT activation. In vitro analysis shows tumour specific differences, as PTEN is likely the dominant mechanism in glioblastoma multiforme (Sano et al, 1999) and prostate cancer (Davies et al, 2002); in breast cancer, HER-2/neu is likely the upstream activator (Zhou et al, 2000). In pancreatic cancer, Matsumoto et al (2002) recently demonstrated that neither mutation of K-ras or PTEN is associated with activation of AKT, though HER-2/neu signalling was not investigated. Despite these in vitro studies, there is little information on which signalling pathway is present in human tumours.

Therefore, we sought to determine whether AKT is activated in the tumours of patients with pancreatic cancer, whether this is associated with HER-2/neu overexpression, and the biologic consequence of these events.

\section{MATERIALS AND METHODS}

\section{Materials}

Cell culture supplies and media were purchased from Becton Dickinson (San Diego, CA, USA) and Gibco/BRL Life Technologies (Gaithersburg, MD, USA), respectively. Mouse monoclonal antibody to HER-2/neu for Western blotting was obtained from Oncogene Research Products (San Diego, CA, USA), while the mouse monoclonal antibody to HER-2/neu for immunohistochemistry was obtained from Dako Corp. (Carpinteria, CA, USA). Detection of the cellular level of AKT as well as the activated, phosphorylated form of AKT (phospho-serine-473) was through the use of polyclonal antibodies (Cell Signaling Technology, Beverly, MA, USA). Inhibition of HER-2/neu was performed using trastuzamab (Herceptin ${ }^{\mathbb{B}}$, Genentech, South San Francisco, CA, USA).

\section{Cell culture}

The seven human pancreatic adenocarcinoma cell lines were obtained from the American Type Culture Collection (Rockville, MD, USA). Cells were cultured in appropriate culture medium supplemented with $10 \%$ fetal calf serum, sodium pyruvate, nonessential amino acids, L-glutamine, penicillin/streptomycin antibiotics. Cells were maintained in a humidified incubator containing $10 \% \mathrm{CO}_{2}$ at $37^{\circ} \mathrm{C}$. Cells underwent serum starvation for the $24 \mathrm{~h}$ prior to treatment with trastuzamab.

\section{Western blotting}

Following treatment, cells were harvested and lysed in a buffer containing $150 \mathrm{~mm} \mathrm{NaCl}, 1 \%$ Triton X-100 and $25 \mathrm{~mm}$ Tris $(\mathrm{pH}$ 7.5). Debris was sedimented by centrifugation for $5 \mathrm{~min}$ at $12000 \mathrm{~g}$, and the supernatants were solubilised for $5 \mathrm{~min}$ at $100^{\circ} \mathrm{C}$ in Laemmli's sodium dodecyl sulphate-polyacrylamide gel electrophoresis (SDS-PAGE) sample buffer containing $100 \mathrm{~mm}$ dithiothreitol. Protein concentrations of the lysates were determined with a protein quantitation kit (Bio-Rad Laboratories, Hercules, CA, USA), and $50 \mu \mathrm{g}$ of each sample was separated on a $10 \%$ SDS PAGE gel. Separated polypeptides were then electrophoretically transferred to $0.2-\mathrm{mm}$ nitrocellulose membranes (Schleicher \& Schuell, Keene, NH, USA). Membranes were blocked for $1 \mathrm{~h}$ in a Tris-buffered saline-Tween (TBS-T; $25 \mathrm{~mm}$ Tris, pH 8.0, $150 \mathrm{~mm}$ $\mathrm{NaCl}$, and $0.05 \%$ Tween-20) containing $5 \%\left(\mathrm{w} \mathrm{v}^{-1}\right)$ nonfat dried milk. Blots were then probed overnight with primary antibodies and developed using species-specific secondary antisera. Immunoreactive material was detected by the enhanced chemilumines- cence technique (Amersham, Piscataway, NJ). Relative polypeptide expression was quantified by laser densitometry (Molecular Dynamics, Sunnyvale, CA, USA).

\section{Identification of pancreatic adenocarcinoma specimens for analysis}

We queried the Tumour Registry database of the University of California, Davis Medical Center for the 4-year period of 19972000 and identified 94 cases of adenocarcinoma of the pancreas. These included surgically resected specimens, intraoperative biopsies, and percutaneous biopsies of metastases. Haematoxylin/eosin-stained slides for all specimens were re-reviewed by a single pathologist and 16 cases were subsequently excluded from the analysis because of histology other than pure adenocarcinoma (e.g. adenosquamous), primary tumour location other than pancreas (e.g. ampulla), or insufficient archival tissue for immunohistochemical (IHC) analysis. The remaining 78 tumours of confirmed pancreatic adenocarcinoma serve as the study group and were re-reviewed by the same pathologist to determine the histologic grade (well-differentiated, moderately well differentiated, or poorly differentiated).

\section{Immunohistochemical technique}

From formaldehyde-fixed, paraffin-embedded tumour specimens, fresh representative sections were cut and subsequently rehydrated with xylene and graded alcohols. Antigen retrieval was performed using the microwave technique in $10 \mathrm{~mm}$ citrate buffer, $\mathrm{pH}$ 6. Both anti-HER-2/neu and anti-phospho-AKT antibodies were used at $1: 50$ dilutions. Visualisation was performed using biotinylated horse-antimouse, streptavidin-HRP system (Vector Labs, Inc., Burlingame, CA, USA) followed by $3,3^{\prime}$-diaminobenzidine (Sigma Chemical Co., St Louis, MO, USA). Sections were counterstained with Gill's haematoxylin. Infiltrating ductal carcinoma from a breast cancer specimen was used as a positive control for the HER2/neu IHC, and the LNCaP prostate cancer cell line harbouring a constitutively activated AKT by virtue of a PTEN mutation was used as the positive control for phospho-AKT (pAKT) (Huang et al, 2001).

\section{Scoring of the IHC staining}

Scoring of HER-2/neu expression used standard quantification as follows: 0 for no staining; $1+$, barely perceptible staining not totally encircling the membrane; $2+$, light to moderate staining totally encircling the membrane; and $3+$, moderate to heavy staining totally encircling the membrane (Yamanaka et al, 1993). HER $2 / n e u$ overexpression was classified as $2+$ or $3+$ with $25 \%$ or more cells staining positive (Koeppen et al, 2001). Immunohistochemical staining of tumour specimens for pAKT was performed using a previously reported scoring system (Malik et al, 2002). In brief, specimens were scored as pAKT positive if $>20 \%$ or $=20 \%$ of the tumour cells demonstrated staining at an intensity equal to or greater than that of the positive control. Statistical analysis was performed using $\chi^{2}$ analysis with significance determined by $P<0.05$.

\section{RESULTS}

\section{Levels of HER-2/neu expression and activation of AKT in} pancreatic cancer cell lines in vitro

We determined the level of HER-2/neu and AKT as well as activated AKT (using a phospho-specific antibody) under basal conditions by Western blotting (Figure 1A). The level of HER-2/ neu varied among the cell lines with CAPAN-1 and MIA-PaCa-2 demonstrating the highest level. While AKT levels were fairly 
A
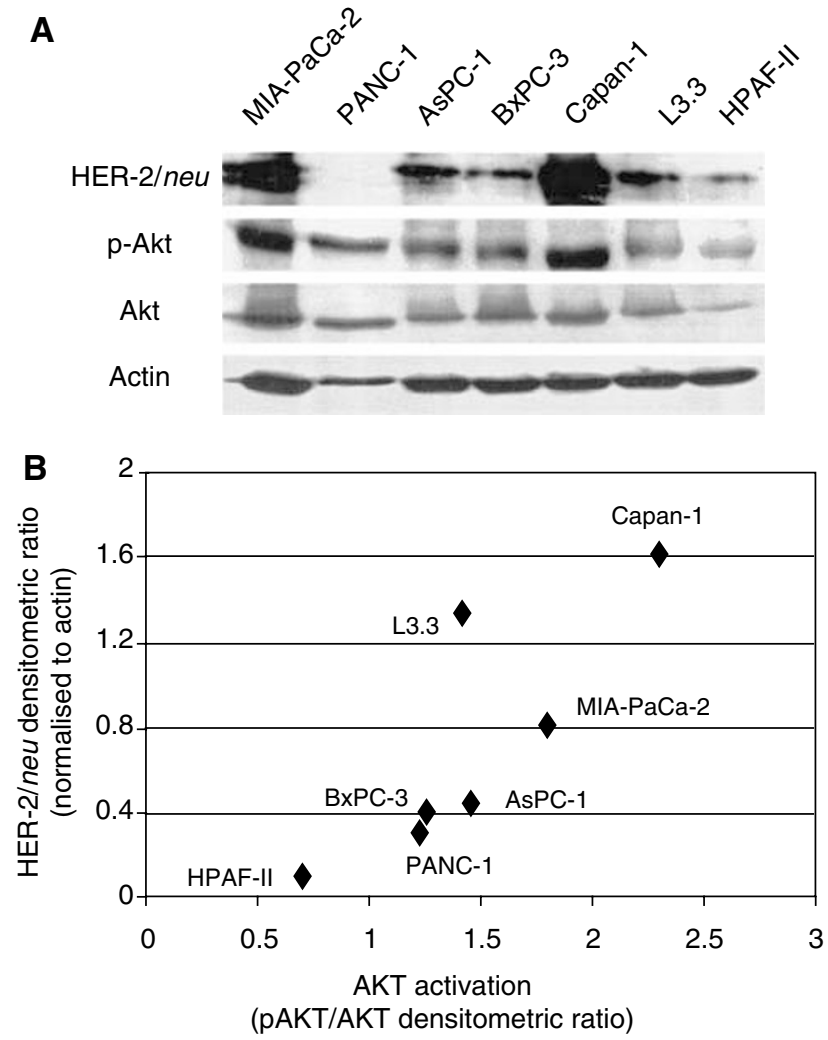

Figure I (A) Levels of HER-2/neu, phospho-AKT (serine-473), and AKT in seven human pancreatic cancer cell lines (MIA-PaCa-2, PANC-I, AsPCI, BxPC-3, L3.3, CAPAN-I and HPAF-II). Western blotting for actin was used as a loading control. (B) Relationship of level of HER-2/neu expression to activation of AKT (ratio of phospho-AKT to AKT) determined following densitometric quantification of Western blot data (coefficient of correlation $=0.82$ ).

similar among most of the cell lines, pAKT was highest in the two cell lines (CAPAN-1 and MIA-PaCa-2) with the highest level of HER-2/neu. When densitometric quantitation of the level of HER$2 /$ neu expression was correlated with relative degree of activation of AKT (the ratio of the pAKT to AKT densitometric evaluation), a linear relationship was observed for six of the seven cell lines (Figure 1B). pAKT was detectable in all cell lines, even the PANC-1 lacking HER-2/neu expression, indicating that upstream signals other than HER-2/neu are involved in the constitutive activation of AKT in these pancreatic cancer cell lines.

\section{Effect of HER-2/neu inhibition on pAKT}

To determine the functional coupling of HER-2/neu to AKT activation, the MIA-PaCa-2 and PANC- 1 cell lines were treated with the neutralising/blocking monoclonal anti-HER-2/neu antibody trastuzamab (Herceptin ${ }^{\mathbb{R}}$ ). MIA-PaCa-2 cells demonstrated a time-dependent decrease in pAKT, beginning at $30 \mathrm{~min}$ and terminating after $2 \mathrm{~h}$ of treatment (Figure 2). This treatment had no effect on total AKT levels, nor any effect on pAKT levels in the PANC-1 cell line that lacks detectable HER-2/neu expression.

\section{Incidence of activated AKT in pancreatic adenocarcinoma tumours}

The 78 tumours represented a mix of localised tumours that underwent resection $(n=35 ; 45 \%)$ and metastatic tumours for which a biopsy was performed for histopathologic diagnosis $(n=43 ; 55 \%)$. Histologic grading demonstrated that almost one-
A

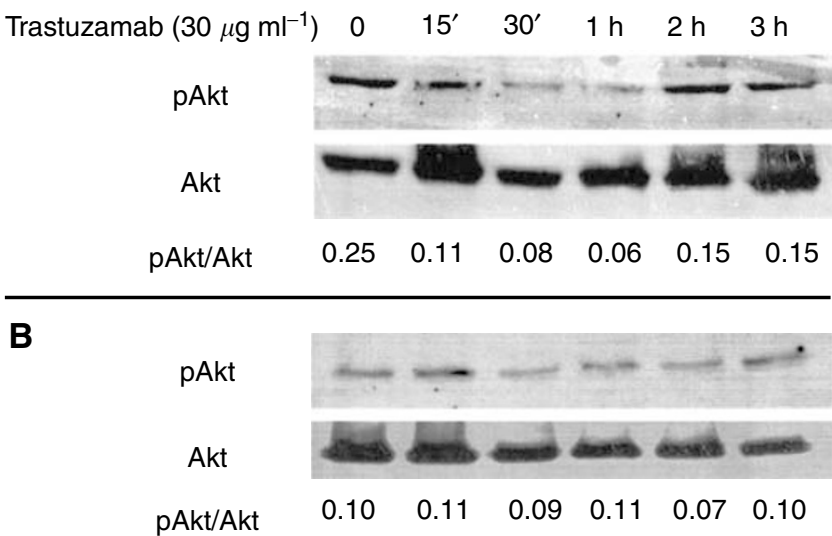

Figure 2 Time-dependent decrease in phospho-AKT following inhibition of HER-2/neu with trastuzamab $(30 \mu \mathrm{g} \mathrm{ml})^{-1}$ in MIA-PaCa-2 (A) or PANCI (B) pancreatic cancer cells. Inhibition was observed following $30 \mathrm{~min}$ of treatment and returned to baseline after $2 \mathrm{~h}$ of treatment. AKT levels are shown for equivalency of loading. Data of densitometric quantification of protein levels are shown below each lane (phospho-AKT normalised to AKT level).

Table I Demographics of the patients $(n=78)$ whose tumors were examined for AKT activation, HER-2/neu overexpression and histologic grade

\begin{tabular}{ll}
\hline Demographics & $\mathbf{n = 7 8}$ \\
\hline Age ( \pm standard deviation) (years) & $65 \pm 12$ \\
Gender (M:F) & $35: 43$ \\
Surgically resected (Y:N) & $35: 43$ \\
& \\
Histologic grade & \\
Well-differentiated & $16(21 \%)$ \\
Moderately well-differentiated & $37(47 \%)$ \\
Poorly differentiated & $25(32 \%)$ \\
AKT activated & $46(59 \%)$ \\
HER-2/neu overexpressed & $52(67 \%)$ \\
\hline
\end{tabular}

$M=$ male; $F=$ female; $Y=$ yes; $N=$ no.

half of tumours were moderately well differentiated, one-fifth were well differentiated, and one-third were poorly differentiated (Table 1). Of these 78 tumour specimens, 46 (59\%) demonstrated activation of AKT by virtue of IHC staining for pAKT (Figure 3). The typical pattern of pAKT staining we observed included both cytoplasmic and nuclear immunostaining. This is consistent with most other reports for malignant cells in which the reported subcellular localisation of pAKT staining is cytoplasmic (Gupta et al, 2002; Perez-Tenorio and Stal, 2002) and nuclear (Dhawan et al, 2002; Itoh et al, 2002). Membrane staining is commonly observed in nontransformed cells and has also been reported in malignant cells though usually as a component of cytoplasmic staining (Yuan et al, 2000).

\section{Incidence of Her-2/neu overexpression and correlation with pAKT in pancreatic adenocarcinoma}

We noted that 51 of the 78 tumours (65\%) demonstrated HER-2/ neu overexpression by immunohistochemistry (Figure 4). We then correlated HER-2/neu overexpression and AKT activation in these 78 pancreatic adenocarcinoma specimens. We found a statistically significant correlation between HER-2/neu overexpression and 

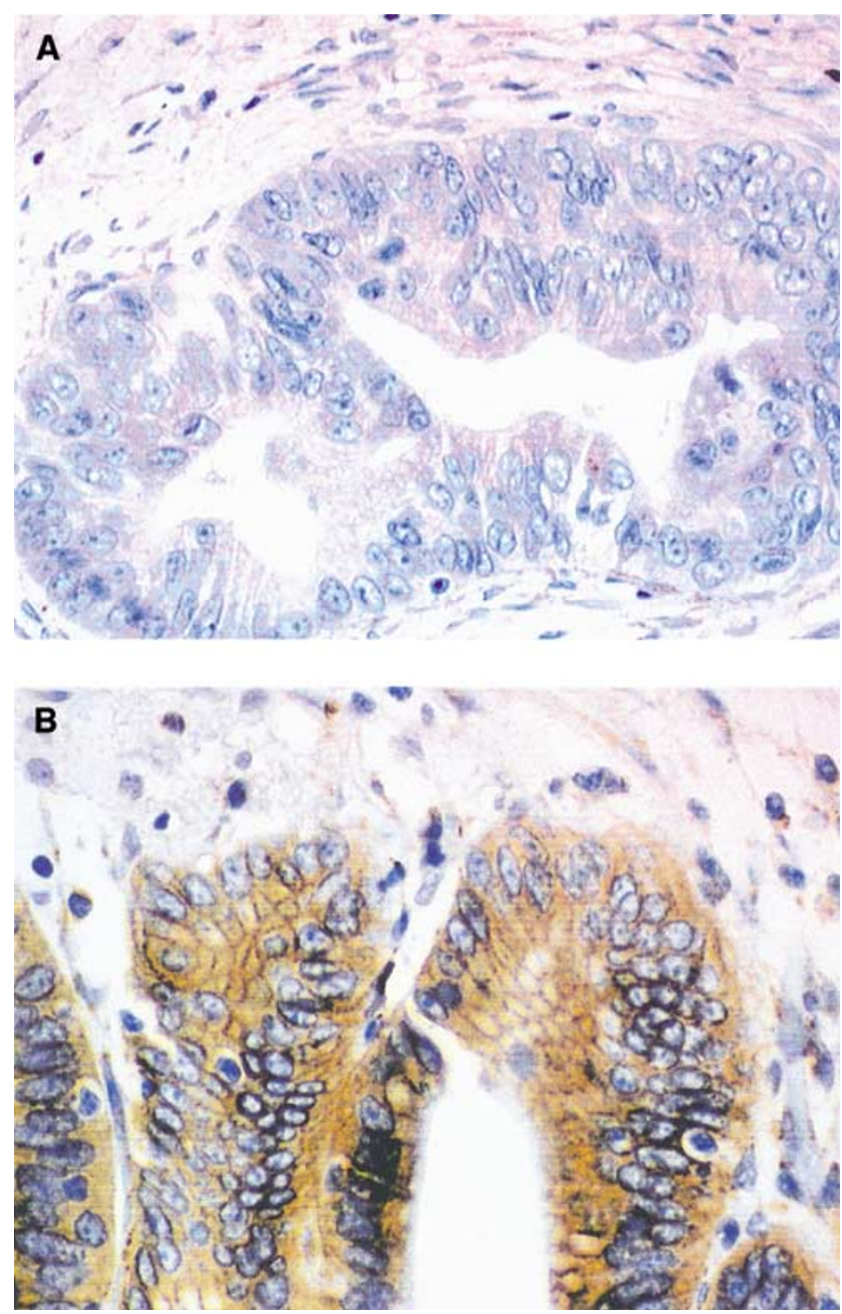

Figure 3 Representative $\mathrm{HC}$ staining for HER-2/neu in pancreatic tumours: $(\mathbf{A})$ tumour without detectable expression and $(\mathbf{B})$ tumour with strong expression including intense membrane staining consistent with overexpression of HER-2/neu ( $\times 40$, original magnification).

activation of AKT (Table 2). Of the 52 HER-2/neu overexpressing tumours, 35 (67\%) demonstrated increased expression of pAKT; of the 26 tumours lacking HER-2/neu expression, 15 (58\%) also lacked pAKT expression. These data would indicate that HER-2/ $n e u$ is one of the major upstream activators of AKT; when HER-2/ $n e u$ is not overexpressed, activation of AKT is uncommon, but when HER-2/neu becomes an overexpression, the majority of tumours will also harbour activated AKT. Of the 46 tumours that were pAKT positive, $35(76 \%)$ demonstrated overexpression of HER-2/neu. AKT can be activated by many upstream receptor tyrosine kinases and this is apparent in the observation that 11 of the $46(24 \%)$ samples demonstrated activated AKT in the absence of HER-2/neu overexpression.

\section{Correlation of activated AKT with histologic grade in pancreatic adenocarcinoma}

For patients with pancreatic adenocarcinoma, the single most important prognostic variable is whether the tumour is localised and can be surgically removed (Takahashi et al, 1997; Hermanek, 1998; Eskelinen and Haglund, 1999; Kedra et al, 2001). Beyond this variable, histologic grade remains one of the most significant prognostic variable, with higher histologic grade (poorly differentiated) being associated with shorter survival. In fact, in studies
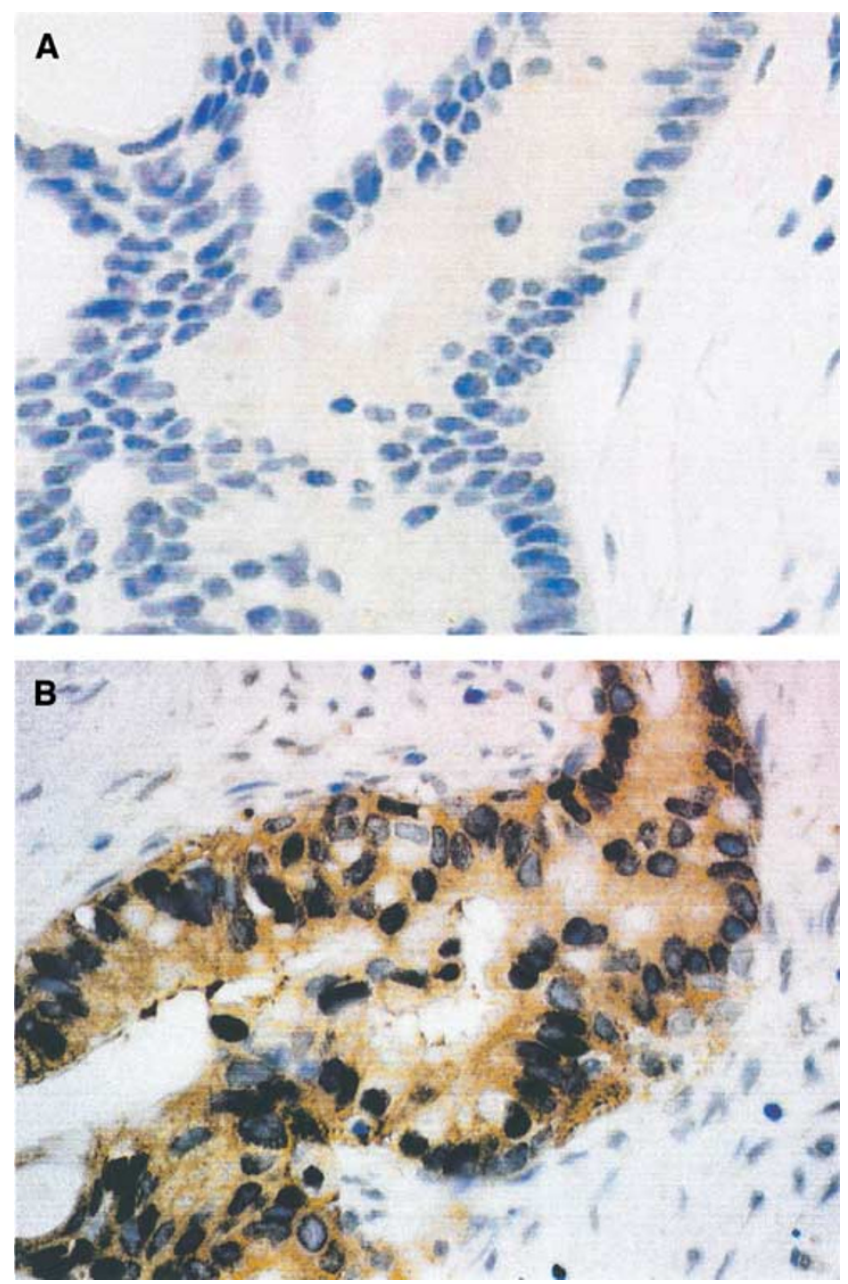

Figure 4 Representative $I H C$ staining for phospho-AKT in pancreatic tumours: $(\mathbf{A})$ tumour without detectable expression and (B) tumour with strong expression of phospho-AKT consistent with activation of AKT. Normal pancreatic ducts do not have detectable phospho-AKT $(\times 40$, original magnification).

Table 2 Correlation between HER-2/neu overexpression and AKT activation $(P=0.015)$

\begin{tabular}{lcc}
\hline & $\begin{array}{c}\text { HER-2/neu } \\
\text { negative }\end{array}$ & $\begin{array}{c}\text { HER-2/neu } \\
\text { overexpressing }\end{array}$ \\
\hline Phospho-AKT negative & 15 & 17 \\
Phospho-AKT positive & 11 & 35 \\
\hline
\end{tabular}

of mixed populations of localised and metastatic pancreatic cancer, histologic grade is the most significant predictor of survival (Takahashi et al, 1997). Survival analysis was not possible in our study population because of two reasons: (1) the patient sample included all stages of pancreatic cancer but with subsets too small for meaningful analysis and (2) survival end points were not consistently available as a significant fraction were referred to UC Davis Medical Center for either the surgical procedure or treatment recommendation, but follow-up was provided by an outside physician. Therefore, we correlated AKT activation with the histologic grade as a surrogate marker of tumour biology. We observed a significant correlation between the presence of activated AKT and higher histologic grade (Table 3). Among the 16 well-differentiated tumours, only six $(38 \%)$ were pAKT positive, 
Table 3 Correlation between AKT activation and histologic grade $(P=0.047)$

\begin{tabular}{lcc}
\hline & $\begin{array}{c}\text { Phospho-AKT } \\
\text { negative }\end{array}$ & $\begin{array}{c}\text { Phospho-AKT } \\
\text { positive }\end{array}$ \\
\hline Well differentiated & 10 & 6 \\
Moderately well differentiated & 16 & 21 \\
Poorly differentiated & 6 & 19 \\
\hline
\end{tabular}

while among the 25 poorly differentiated tumours, 19 (76\%) were pAKT positive $(P=0.047)$. These data suggest that activated AKT correlates with a marker of aggressive tumour biology in pancreatic cancer.

\section{DISCUSSION}

There has been increasing evidence that AKT is activated in many different cancer types and that this signalling pathway confers a potent survival signal that relates to chemo- and radioresistance (Brognard et al, 2001; West et al, 2002). Furthermore, early studies have demonstrated that inhibition of AKT sensitises tumours to the apoptotic effect of both chemo- and radiotherapy. The reported incidence of AKT activation varies from $26 \%$ in prostate cancer (Malik et al, 2002) to $66 \%$ in melanoma (Dhawan et al, 2002), and aerodigestive squamous cell carcinoma (Gupta et al, 2002). Our data demonstrate that AKT is activated in $59 \%$ of the pancreatic adenocarcinoma tumours studied, placing it near the top of tumours that have been reported to harbour AKT activation. Furthermore, AKT activation correlated with the histologic grade, which remains one of the most significant prognostic variables in this cancer, surpassing tumour size or nodal status for predicting survival (Takahashi et al, 1997; Eskelinen and Haglund, 1999; Luttges et al, 2000; Kedra et al, 2001).

Although mutation and/or deletion of PTEN can lead to constitutive activation of $\mathrm{AKT}$, it is unknown whether this is the dominant mechanism in tumours other than specific subtypes, such as glioblastome multiforme (Sano et al, 1999). Preliminary in vitro evidence implicates HER-2/neu as a potential mediator of AKT activation with initiation of an antiapoptotic signal (Zhou et al, 2001). Furthermore, Zhou et al (2000) examined 10 breast cancer specimens overexpressing HER-2/neu; seven demonstrated AKT activation. Similarly, only two-thirds of the HER-2/neu overexpressing pancreatic tumours demonstrated AKT activation; this suggests that some minor fraction of tumours have an additional defect in the signalling pathway from HER-2/neu to $\mathrm{AKT}$ that prevents AKT activation despite HER-2/neu overexpression. Therefore, if targeted HER-2/neu therapy using agents such as trastuzamab is to be employed in pancreatic cancer, and the mechanism of this therapy is mediated by AKT, then it would be anticipated that only tumours demonstrating significant AKT activation would respond. This may explain the mixed results that have been observed in the early clinical trials of trastuzamab in pancreatic cancer (Iannitti et al, 2003).

It is also clear that additional upstream mediators other than HER-2/neu can activate AKT. In both the examination of the cell lines in vitro as well as the tumour specimens, activated AKT was observed in the absence of HER-2/neu overexpression. There are numerous genetic events in pancreatic cancer that could potentially lead to activation of $\mathrm{AKT}$, including mutations of either K-ras or PTEN, and constitutive activation of a variety of receptor tyrosine kinases. Therefore, it would appear that HER-2/ neu overexpression mediates the activation of AKT in some, but not all, pancreatic cancers that demonstrate constitutive activation of AKT. The downstream effectors of AKT that mediate the biologic events are under active investigation. In vitro data have coupled AKT to the survival pathway of the transcription factor NF- $\kappa \mathrm{B}$. This is particularly interesting given three observations: (1) the frequent constitutive activation of NF- $\kappa \mathrm{B}$ in various cancers including pancreatic tumour specimens (Wang et al, 1999), (2) the nuclear immunostaining pattern of pAKT frequently observed in various tumours (Dhawan et al, 2002; Itoh et al, 2002; Perez-Tenorio and Stal, 2002), and (3) the correlation of pAKT immunostaining and nuclear localisation of the p65 subunit of NF- $\kappa \mathrm{B}$ in melanoma specimens (Dhawan et al, 2002). We have previously shown in the MIA-PaCa- 2 cells that AKT is coupled to NF- $\kappa \mathrm{B}$ activation and alters levels of various members of the apoptosis-regulating BCL-2 family (Fahy et al, 2003).

Inhibition of both HER-2/neu and AKT has been shown to increase the apoptotic sensitivity of pancreatic cancer in vitro and in vivo (Ng et al, 2000, 2001; Buchler et al, 2001; Bondar et al, 2002; Fahy et al, 2003). Interestingly, Buchler et al noted that the MIA$\mathrm{PaCa}-2$ cell line was the most sensitive to the cytotoxic effect of trastuzamab, PANC-1 and AsPC-1 intermediately sensitive and HPAF-II was insensitive. These data closely parallel both the levels of HER-2/neu and AKT activation we noted in Figure 1B. While some may promote therapy targeted at AKT independent of mechanism of AKT activation, the in vitro data demonstrates the highest degree of AKT activation is observed in those cell lines with the highest level of HER-2/neu. Therefore, tumours demonstrating AKT activation by virtue of HER-2/neu overexpression may be the most sensitive to therapy directed at AKT. Although this has not been investigated, research into predicting responses to targeted therapy based on molecular and genetic profiles of individual cancers is actively being pursued.

Although AKT amplification has been previously reported in pancreatic cancer specimens (Ruggeri et al, 1998), gene amplification does not necessarily correlate with increased kinase activity. Using phospho-specific antibodies, we are now able to determine the activated status of specific kinases and determine whether a specific signalling cascade is functional. Constitutive activation of AKT has been observed in various cancers and represents an evolving target for therapy in cancer. Our data demonstrate that pancreatic cancer may share some of the same biologic characteristics as breast cancer with regards to HER-2/neu and AKT signalling, generating some enthusiasm for targeted HER-2/ $n e u$ or AKT therapy in this lethal malignancy.

\section{ACKNOWLEDGEMENTS}

This research was supported in part by grants from the Lustgarten Foundation (LF01-032) and the University of California Cancer Research Commission.

\section{REFERENCES}

Bardeesy N, DePinho RA (2002) Pancreatic cancer biology and genetics. Nat Rev Cancer 2: 897-909

Bondar VM, Sweeney-Gotsch B, Andreeff M, Mills GB, McConkey DJ (2002) Inhibition of the phosphatidylinositol $3^{\prime}$-kinase-AKT pathway induces apoptosis in pancreatic carcinoma cells in vitro and in vivo. Mol Cancer Ther 1: 989-997

Brognard J, Clark AS, Ni Y, Dennis PA (2001) Akt/protein kinase B is constitutively active in non-small cell lung cancer cells and promotes cellular survival and resistance to chemotherapy and radiation. Cancer Res 61: 3986-3997

Buchler P, Reber HA, Buchler MC, Roth MA, Buchler MW, Friess H, Isacoff WH, Hines OJ (2001) Therapy for pancreatic cancer with a recombinant 
humanized anti-HER2 antibody (herceptin). J Gastrointest Surg 5: $139-146$

Davies MA, Kim SJ, Parikh NU, Dong Z, Bucana CD, Gallick GE (2002) Adenoviral-mediated expression of MMAC/PTEN inhibits proliferation and metastasis of human prostate cancer cells. Clin Cancer Res 8: $1904-1914$

Dhawan P, Singh AB, Ellis DL, Richmond A (2002) Constitutive activation of Akt/protein kinase B in melanoma leads to up-regulation of nuclear factor-kappaB and tumour progression. Cancer Res 62: 7335-7342

Eskelinen MJ, Haglund UH (1999) Prognosis of human pancreatic adenocarcinoma: review of clinical and histopathological variables and possible uses of new molecular methods. Eur J Surg 165: 292 - 306

Fahy BN, Schlieman M, Virudachalam S, Bold RJ (2003) AKT inhibition is associated with chemosensitisation in the pancreatic cancer cell line MIA-PaCa-2. Br J Cancer 89: 391 - 397

Guerrero S, Casanova I, Farre L, Mazo A, Capella G, Mangues R (2000) $\mathrm{K}$-ras codon 12 mutation induces higher level of resistance to apoptosis and predisposition to anchorage-independent growth than codon 13 mutation or proto-oncogene overexpression. Cancer Res 60: 6750-6756

Gupta AK, McKenna WG, Weber CN, Feldman MD, Goldsmith JD, Mick R, Machtay M, Rosenthal DI, Bakanauskas VI, Cerniglia GJ, Bernhard EJ, Weber RS, Muschel RJ (2002) Local recurrence in head and neck cancer: relationship to radiation resistance and signal transduction. Clin Cancer Res 8: $885-892$

Haas-Kogan D, Shalev N, Wong M, Mills G, Yount G, Stokoe D (1998) Protein kinase B (PKB/Akt) activity is elevated in glioblastoma cells due to mutation of the tumor suppressor PTEN/MMAC. Curr Biol 8: $1195-1198$

Heinemann V (2002) Present and future treatment of pancreatic cancer. Semin Oncol 29(3 Suppl 9): 23-31

Hermanek P (1998) Pathology and biology of pancreatic ductal adenocarcinoma. Langenbecks Arch Surg 383: 116-120

Huang H, Cheville JC, Pan Y, Roche PC, Schmidt LJ, Tindall DJ (2001) PTEN induces chemosensitivity in PTEN-mutated prostate cancer cells by suppression of Bcl-2 expression. J Biol Chem 276: $38830-38836$

Iannitti DA, Coburn N, Cioffi W, Safran H, Steinoff M, Ramanathan R, Schwartz J (2003) Trastuzumab (Herceptin) and gemcitabine (Gemzar) for metastatic pancreas cancers that overexpress HER2/neu. I Gastrointest Surg 7: 294

Itoh $\mathrm{N}$, Semba S, Ito $\mathrm{M}$, Takeda $\mathrm{H}$, Kawata S, Yamakawa M (2002) Phosphorylation of Akt/PKB is required for suppression of cancer cell apoptosis and tumour progression in human colorectal carcinoma. Cancer 94: 3127-3134

Kedra B, Popiela T, Sierzega M, Precht A (2001) Prognostic factors of longterm survival after resective procedures for pancreatic cancer. Hepatogastroenterology 48: 1762-1766

Koeppen HKW, Wright BD, Burt AD, Quirke P (2001) Overexpression of HER2/neu in solid tumours: an immunohistochemical survey. Histopathology 38: $96-104$

Luttges J, Schemm S Vogel I, Hedderich J, Kremer B, Kloppel G (2000) The grade of pancreatic ductal carcinoma is an independent prognostic factor and is superior to the immunohistochemical assessment of proliferation. J Pathol 191: 154-161

Malik SN, Brattain M, Ghosh PM, Troyer DA, Prihoda T, Bedolla R, Kreisberg JI (2002) Immunohistochemical demonstration of phospho-Akt in high Gleason grade prostate cancer. Clin Cancer Res 8: $1168-1171$

Matsumoto J, Kaneda M, Tada M, Hamada J, Okushiba S, Kondo S, Katoh $\mathrm{H}$, Moriuchi T (2002) Differential mechanisms of constitutive Akt/PKB activation and its influence on gene expression in pancreatic cancer cells. Jpn J Cancer Res 93: 1317-1326

Ng SSW, Tsao MS, Chow S, Medley DW (2000) Inhibition of phosphatidylinositide 3-kinase enhances gemcitabine-induced apoptosis in human pancreatic cancer cells. Cancer Res 60: 5451-5455

Ng SS, Tsao MS, Nicklee T, Medley DW (2001) Wortmannin inhibits pkb/ akt phosphorylation and promotes gemcitabine antitumor activity in orthotopic human pancreatic cancer xenografts in immunodeficient mice. Clin Cancer Res 7: 3269-3275

Perez-Tenorio G, Stal O (2002) Activation of AKT/PKB in breast cancer predicts a worse outcome among endocrine treated patients. Br J Cancer 86: $540-545$

Perugini RA, McDade TP, Vittimberga FJ, Gallery MP (2000) Pancreatic cancer cell proliferation is phosphatidylinositol 3-kinase dependent. J Surg Res 90: 39-44

Ruggeri BA, Huang L, Wood M, Cheng JQ, Testa JR (1998) Amplification and overexpression of the AKT2 oncogene in a subset of human pancreatic ductal adenocarcinomas. Mol Carcinogen 21: 81-86

Samatar AA, Wang L, Mirza A, Koseoglu S, Liu S, Kumar CC (2002) Transforming growth factor-beta 2 is a transcriptional target for Akt/ protein kinase B via forkhead transcription factor. J Biol Chem 277: $28118-28126$

Sano T, Lin H, Chen X, Langford LA, Koul D, Bondy ML, Hess KR, Myers JN, Hong YK, Yung WK, Steck PA (1999) Differential expression of MMAC/PTEN in glioblastoma multiforme: relationship to localization and prognosis. Cancer Res 59: $1820-1824$

Takahashi T, Niino N, Ishikura H, Okushiba S, Dohke M, Katoh H (1997) Predictive factors for long-term survival in patients with pancreatic carcinoma. Hepatogastroenterology 44: 1463-1468

Tang D, Okada H, Ruland J, Liu L, Stambolic V, Mak TW, Ingram AJ (2001) Akt is activated in response to an apoptotic signal. J Biol Chem 276: 30461 - 30466

Vivanco I, Sawyers CL (2002) The phosphatidylinositol 3-kinase AKT pathway in human cancer. Nat Rev Cancer 2: 489-501

Wang W, Abbruzzese JL, Evans DB, Larry L, Cleary KR, Chiao PJ (1999) The nuclear factor-kappa B RelA transcription factor is constitutively activated in human pancreatic adenocarcinoma cells. Clin Cancer Res 5: $119-127$

West KA, Sianna-Castillo S, Dennis PA (2002) Activation of the PI3K/Akt pathway and chemotherapeutic resistance. Drug Resist Update 5: 234-248

Xue L, Murray JH, Tolkovsky AM (2000) The ras/phosphatidylinositol 3kinase and ras/ERK pathways function as independent survival modules each of which inhibits a distinct apoptotic signaling pathway in sympathetic neurons. J Biol Chem 275: 8817-8824

Yamanaka Y, Friess H, Kobrin M, Buchler M (1993) Overexpression of HER2/neu oncogene in human pancreatic carcinoma. Hum Pathol 24: $1127-1134$

Yao Z, Okabayashi Y, Yutsudo Y, Kitamura T, Ogawa W, Kasuga M (2002) Role of Akt in growth and survival of PANC-1 pancreatic cancer cells. Pancreas 24: $42-43$

Yuan ZQ, Sun M, Feldman RI, Wang G, Ma X, Jiang C, Coppola D, Nicosia SV, Cheng JQ (2000) Frequent activation of AKT2 and induction of apoptosis by inhibition of phosphoinositide-3-OH kinase/Akt pathway in human ovarian cancer. Oncogene 19: 2324-2330

Zhou BP, Hu MC, Miller SA, Yu Z, Xia W, Lin SY, Hung MC (2000) HER-2/ neu blocks tumour necrosis factor-induced apoptosis via the Akt/NFkappaB pathway. J Biol Chem 275: 8027-8031

Zhou BP, Liao Y, Xia W, Zou Y, Spohn B, Hung MC (2001) HER-2/neu induces p53 ubiquitination via Akt-mediated MDM2 phosphorylation. Nat Cell Biol 3: 973-982 